\title{
The Effects of Elastic Ankle Taping on Static and Dynamic Postural Control in Individuals With Chronic Ankle Instability
}

\author{
Jin-seok Lim¹, PT, BPT, Seo-hyun Kim¹, PT, BPT, Il-young Moon¹, PT, MSc, Chung-hwi Yi², PT, PhD \\ ${ }^{1}$ Department of Physical Therapy, The Graduate School, Yonsei University, ${ }^{2}$ Department of Physical Therapy, College of Software and Digital \\ Healthcare Convergence, Yonsei University, Wonju, Korea
}

\author{
Article Info \\ Received July 5, 2021 \\ Revised July 13, 2021 \\ Accepted July 13, 2021 \\ Corresponding Author \\ Chung-hwi Yi \\ E-mail: pteagle@yonsei.ac.kr \\ https://orcid.org/0000-0003-2554-8083
}

\section{Key Words}

Ankle joint

Dynamic tape

Joint instability

Kinesio tape

Postural control
Background: Postural control deficit is a major characteristic in patients with chronic ankle instability (CAl). Elastic ankle tapings are commonly used to facilitate postural control in patients with CAl as well as prevent relapse of a lateral ankle sprain. However, equivocal evidence exists concerning the effect of elastic ankle taping on postural control.

Objects: This study aimed to evaluate the effects of elastic ankle tapings using kinesio taping (KT) and dynamic taping (DT) on static and dynamic postural control in patients with CAI.

Methods: Fifteen subjects with CAl were participated in this study. The participants performed tests under three conditions (barefoot, KT, and DT). Static postural control was evaluated using the one-leg standing test (OLST) and dynamic postural control using the modified Star Excursion Balance Test (mSEBT). One-way repeated-measures analysis of variance was used to compare center of pressure (COP) data and normalized mSEBT reach distances among the three conditions (with $\alpha=0.05$ ).

Results: The CoP parameters (path length, ellipse area, and mean velocity) of the OLST significantly decreased on applying KT and DT compared with those when barefoot. The normalized reach distances in the anteromedial (AM), medial (M), and posteromedial (PM) directions of the mSEBT significantly increased with DT compared to that in the control condition. Further, the higher reach distances with KT compared with those in the control condition were obtained in the $\mathrm{M}$ and PM directions of the MSEBT. No significant differences were identified in any of the OLST and SEBT parameters between the two different taping applications.

Conclusion: KT and DT improved static postural control during the OLST compared with the control condition. Moreover, these tapes improved dynamic postural control during the mSEBT compared to the control. Therefore, elastic ankle tapings are useful prophylactic devices for the prevention and treatment of ankle sprain in people with CAI.

\section{INTRODUCTION}

Lateral ankle sprain (LAS) is a major musculoskeletal injury that occurs both in the general and athletic population [1]. LAS potentially leads to structural damages to the ligaments, nerves, and musculotendinous structures around the ankle joint [2]. They can impede both activities of daily living and athletic participation. These damages increase the possibility of relapse by up to $80 \%$ and cause chronic ankle instability (CAI) [3]. CAI is a complicated syndrome that is characterized by various insufficiencies, including laxity of the lateral ligament, muscle weakness, diminished joint proprioception, impaired neuromuscular control, and frequent episodes of giving way [4].
A major characteristic of CAI is postural control deficit [5]. Sensory and motor responses can affect postural control [6]. Moreover, these factors potentially affect the range of motion, muscle strength, and coordination [6]. Due to the importance of postural control in activities of daily living, some studies have investigated various treatment strategies in order to improve postural control, such as taping, bracing, orthotics [7-10].

Kinesio taping (KT) is a commonly used elastic tape in sports and rehabilitation [11]. KT reportedly serves as a prophylactic treatment against rapid hypersupination of ankle joints while providing more ease than athletic tapes because of its skinmimicking property $[12,13]$. Dynamic taping (DT), another type of elastic tape, is a biomechanical tape. DT has certain 
properties that include deceleration, load absorption, and assistance with movement as its primary mode of action. This is due to DT's elasticity of $>200 \%$ of the elongation capacity and multidirectional nature, in which elongation occurs in all directions [14].

Equivocal evidence has been yielded by several studies that have examined the effects of KT on ankle joint stability in CAI patients $[10,12,15-18]$. Some studies have found that KT potentially decreases postural sway, thus providing reinforcement of ankle stability during functional tasks, such as the Star Excursion Balance Test (SEBT) and Y balance test $[10,19,20]$. Moreover, other studies evaluating postural control using the single legged-balance test and quiet standing have demonstrated that KT noticeably enhances postural control in individuals with CAI [21-23]. However, some studies have found no improvement in performance during the SEBT with KT [24-27]. Shields et al. [21] found that the elastic tape could not improve the sway of the center of pressure (CoP) during the single legged-balance test.

To the best of our knowledge, research regarding the effect of DT application in patients with CAI is lacking. Moreover, studies simultaneously investigating and comparing the effects of KT and DT on postural control in individuals with CAI are limited. Thus, this study aimed to evaluate the effects of ankle tapings, namely, KT and DT, on static and dynamic postural control in patients with CAI. The one-leg standing test (OLST) and modified SEBT (mSEBT) were used to measure static and dynamic postural control, respectively. We hypothesized that both the KT and DT applications improve postural stability compared with the control condition (barefoot).

\section{MATERIALS AND METHODS}

\section{Participants}

At least 7 participants were required in this study after considering a power of 0.80 , level of 0.05 in repeated measures analysis of variance (ANOVA), and effect size of 0.58 obtained in a preliminary study. Fifteen patients with unilateral CAI between the ages of 21 and 26 years participated in this study. We determined the inclusion and exclusion criteria for patients with CAI using the International Ankle Consortium guidelines [28]. The inclusion criteria were as follows: (1) a history of at least one specific ankle sprain that occurred more than 12 months before this study, (2) having perceived the feeling of instability and/or ankle giving way at least twice within the last 6 months, (3) a Cumberland Ankle Instability Tool score <24, and (4) an Activities of Daily Living Subscale score $<90 \%$ and Sport Subscale of the Foot and Ankle Ability Measure score $<80 \%$. Table 1 shows the characteristics of the participants in this study. Individuals who had undergone any lower-extremity surgery and those who experienced any nervous and vestibular system problem or tape allergy were excluded from the study. The experiment was conducted after all participants had read and signed an informed consent form. This study was approved by the Institutional Review Board of Yonsei University (IRB number: 1041849-202104-BM-055-01).

\section{Instrumentation}

Twenty-second OLST measurement was performed using a $2,122 \times 605 \times 21-\mathrm{mm}$ (length $\times$ width $\times$ height $)$ Zebris pressure measurement plate (FDM- 2 system; Zebris Medical GmbH, Isny, Germany) [29]. CoP data were sampled at $100 \mathrm{~Hz}$. The CoP path length (LENGTH, mm), mean sway area of the 95\% confidence ellipse area (AREA, $\mathrm{mm}^{2}$ ), and mean velocity of the CoP displacements (VEL, $\mathrm{mm} / \mathrm{s}$ ) were measured during the OLST [29]. Data were processed using MyoResearch software (MyoResearch 3.16; Noraxon, Scottsdale, AZ, USA).

\section{Taping Types and Techniques}

In this study, we used two types of tapes: (1) a 5-cm wide KT (Towatek Korea, Guri, Korea) and (2) a 5-cm wide DT (Dynamic Tape, Port Vila, Vanuatu). The same investigator applied the taping techniques to all participants.

In the KT ankle-taping technique, three strips of KT, one Istripped tape, and two Y stripped tapes were used in that order (Figure 1). First, the I-stripped tape was attached from the

Table 1. Participant demographics ( $N=15)$

\begin{tabular}{lc}
\hline \multicolumn{1}{c}{ Characteristics } & Values \\
\hline Age $(\mathrm{y})$ & $23.5 \pm 1.7$ \\
Sex (male:female) & $9: 6$ \\
Height (cm) & $178.1 \pm 7.6$ \\
Weight (kg) & $84.9 \pm 13.6$ \\
No. of previous ankle sprains & $6.5 \pm 2.6$ \\
CAIT score & $13.3 \pm 4.3$ \\
FAAM, ADL $(\%)$ & $76.0 \pm 5.7$ \\
FAAM, Sport $(\%)$ & $62.1 \pm 3.1$ \\
\hline
\end{tabular}

Values are presented as number only or mean \pm standard deviation. CAIT, cumberland ankle instability scale; FAAM, foot and ankle ability measure; $A D L$, activities of daily living. 


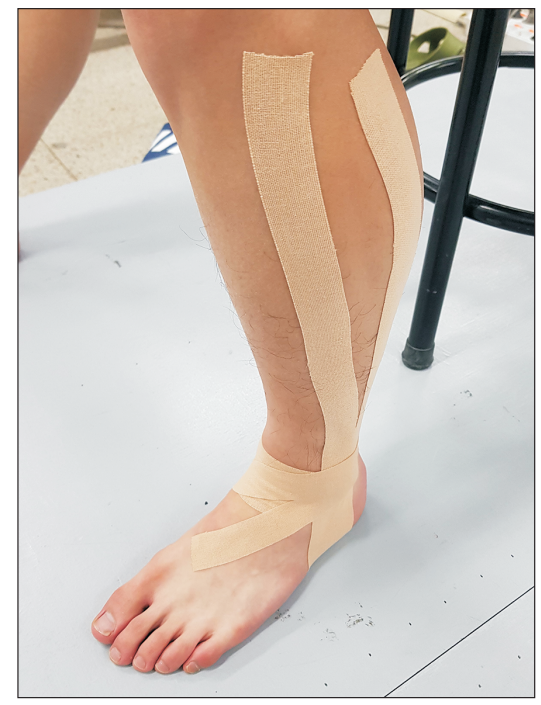

Figure 1. Kinesio ankle taping technique.

lateral to the medial malleolus. Thereafter, the long Y stripped tape was attached to the lateral side of the ankle joint and placed in such a way that the tails stretched along the leg. The origin of the short $Y$ stripped tape was placed on the back of the heel and stretched towards the hallux [11].

In the DT ankle taping technique, single strips with different lengths were used according to the participants' anthropometrical characteristics (Figure 2). First, the participants' starting position was plantar flexion and eversion. The DT was attached and anchored at the head of the first metatarsal joint and proceeded down to the top of the calcaneus posteriorly. Subsequently, the DT was swept around the inside of the heel and attached from the lateral side of the foot. The anchor was attached at one-third of the medial part of the tibia [14].

\section{Procedures}

The participants attended one test session, and static postural control was evaluated using the 20-second OLST on a force plate. Subsequently, dynamic postural control was assessed using the mSEBT. The order of the 3 experimental conditions (bare foot, KT, and DT) was randomized using the random number generator in Excel software (Microsoft corp., Remond, WA, USA).

The first task was the OLST, and the participants were instructed to look forward and place their hands on their hips and hold their contralateral leg at approximately $30^{\circ}$ hip flexion and $90^{\circ}$ knee flexion. Five practice trials were conducted to prevent the "learning effect." The subjects maintained this

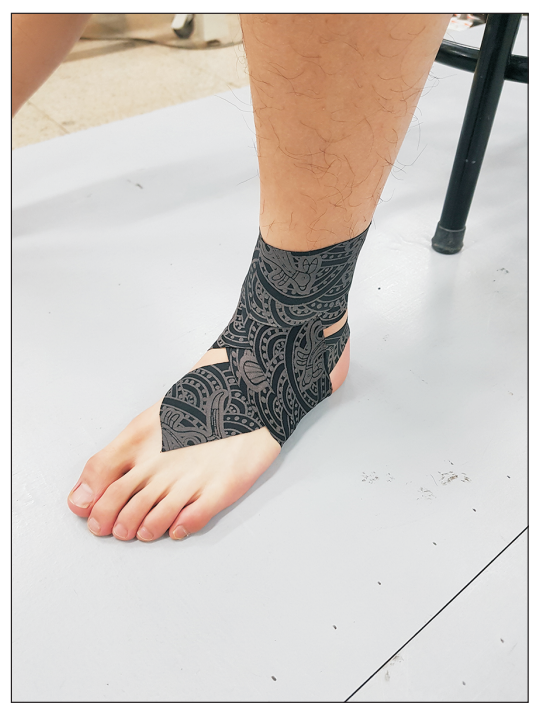

Figure 2. Dynamic ankle taping technique.

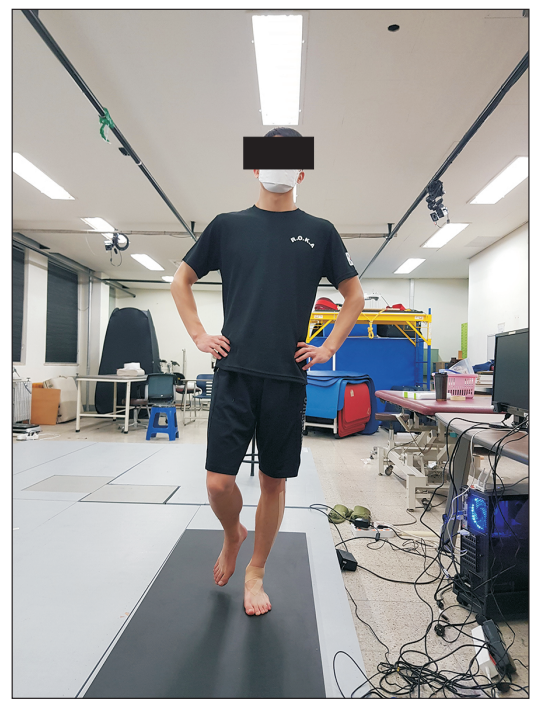

Figure 3. One-leg standing test position on the plate form.

position for 20 seconds. If the contralateral leg touched the floor, the trials were repeated. The participants rested for 30 seconds between trials to minimize the effect of fatigue. Three successful trials were used in the statistical analysis (Figure 3).

After the first task was completed, the participants took a 5-minute rest and subsequently proceeded with the second task, namely, the mSEBT. The SEBT has been recommended as a reliable test for determining postural control deficit in patients with CAI [30]. The modified version, mSEBT, was conducted in 3 directions at $45^{\circ}$ to each other from the center, and the participants placed the test foot of their stance leg in the middle of the testing grid. They were instructed to stretch 
out the opposite leg maximally in the anteromedial (AM), medial (M), and posteromedial (PM) directions (Figure 4) [31].

As their hands remained on their hips, the most distal part of their reaching foot contacted the ground and returned to a double-leg stance position. If the foot of the tested leg did not remain in contact with the ground, the trial was repeated. In order to prevent the "learning effect," 5 practice trials were conducted in each direction prior to the test session [32]. Reach distances were recorded by measuring the distance from the center to the mark point using a tape measure $(\mathrm{cm})$. Thereafter, 3 successful trials in 3 directions were used in the statistical analysis. Rest periods of 30 seconds and 2 minutes were applied between each direction and between trials to minimize the effect of fatigue, respectively [33].

\section{Data Processing and Statistical Analysis}

All the parameters recorded during the OLSTs and SEBTs were calculated for each condition. The mean value of the 3 successful trials in each condition was assessed in the statisti-
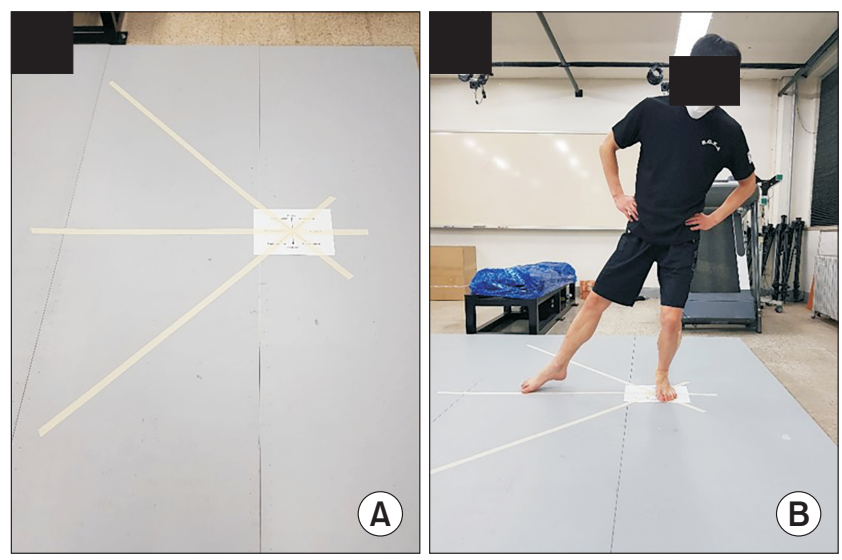

Figure 4. Modified Star Excursion Balance Test. (A) Three directions. (B) Performance in the medial direction. cal analysis. The mean of the 3 successful reach distances were normalized to each participant's leg length [34].

The Shapiro-Wilk statistical test was used to confirm the normality of the data distribution. One-way ANOVA was used to compare CoP data and normalized mSEBT reach distances among the three conditions (barefoot, KT, and DT). The $\alpha$ level of significance was set at 0.05 . Post-hoc pairwise comparisons (Bonferroni procedure) were used to determine where the differences existed (with $\alpha=0.05 / 3=0.017$ ). All statistical analyses were performed using IBM SPSS software (version 25; IBM Corp., Armonk, NY, USA).

\section{RESULTS}

Table 2 displays the means and standard deviations of all CoP parameters as well as the normalized reach distances in 3 directions for the 3 conditions. Table 3 shows the mean differences and p-values of the post-hoc pairwise comparisons of the 3 conditions.

On the OLST, participants exhibited different static postural control among the 3 conditions. On comparing CoP parameters among the 3 conditions, the mean values decreased in the following order: barefoot, KT, and DT. LENGTHs, AREAs, and VELs significantly differed between KT application and barefoot $(\mathrm{p}<0.05)$. Moreover, all CoP parameters were significantly different between KT and barefoot $(\mathrm{p}<0.05)$. Nevertheless, no significant differences between KT and DT were observed ( $p>$ 0.05).

As regards normalized reach distances, significant differences were identified among the 3 conditions in all directions. The post-hoc analysis revealed that the normalized reach distances in the M and PM directions, but not in the AM direction, were significantly different between barefoot and KT ( $p>0.05)$. On

Table 2. Means and standard deviations of CoP parameters and the normalized reach distances in 3 conditions

\begin{tabular}{lccc}
\hline & & Conditions & \\
\cline { 2 - 4 } & Barefoot & Kinesio taping & Dynamic taping \\
\hline CoP parameters & & & $797.5 \pm 204.2$ \\
CoP path length (mm) & $907.8 \pm 256.9$ & $822.9 \pm 216.1$ & $984.2 \pm 300.8$ \\
Ellipse area $\left(\mathrm{mm}^{2}\right)$ & $1,251.0 \pm 516.1$ & $1,017.6 \pm 386.1$ & $39.6 \pm 9.7$ \\
Mean velocity (mm/s) & $45.5 \pm 12.7$ & $41.1 \pm 10.9$ & $81.2 \pm 9.0$ \\
Reach directions $(\mathrm{cm})$ & & & $81.2 \pm 7.9$ \\
Anteromedial & $77.7 \pm 9.0$ & $80.0 \pm 8.8$ & $84.4 \pm 8.3$ \\
Medial & $76.3 \pm 6.3$ & $80.0 \pm 7.9$ & $83.3 \pm 8.5$ \\
Posteromedial & $79.1 \pm 7.9$ & & \\
\hline
\end{tabular}

CoP, center of pressure. 
Table 3. Post-hoc pairwise comparisons of CoP parameters and the normalized reach distances in 3 conditions

\begin{tabular}{|c|c|c|c|c|c|c|}
\hline & \multicolumn{6}{|c|}{ Conditions } \\
\hline & \multicolumn{2}{|c|}{ Barefoot - KT } & \multicolumn{2}{|c|}{ Barefoot - DT } & \multicolumn{2}{|c|}{ KT - DT } \\
\hline & MD & $\mathrm{p}$-value & MD & $\mathrm{p}$-value & MD & $\mathrm{p}$-value \\
\hline \multicolumn{7}{|l|}{ CoP parameters } \\
\hline CoP path length & 84.889 & $0.029 *$ & 110.289 & $0.019 *$ & 25.400 & 0.320 \\
\hline Ellipse area & 233.400 & $0.049 *$ & 266.778 & $0.017^{*}$ & 33.378 & 0.610 \\
\hline Mean velocity & 4.378 & $0.028 *$ & 5.867 & $0.012^{*}$ & 1.489 & 0.255 \\
\hline \multicolumn{7}{|l|}{ Reach directions } \\
\hline Anteromedial & -2.312 & 0.066 & -3.532 & $0.006^{*}$ & -1.219 & 0.390 \\
\hline Medial & -3.622 & $0.012^{*}$ & -4.965 & $0.001 *$ & -1.343 & 0.262 \\
\hline Posteromedial & -4.185 & $0.002^{*}$ & -5.292 & $0.003^{*}$ & -1.107 & 0.436 \\
\hline
\end{tabular}

KT, kinesio taping; DT, dynamic taping; MD, mean difference; CoP, center of pressure. ${ }^{*} p<0.05$.

the other hand, DT application yielded longer reach distances than the other 2 conditions. In addition, all reach distances in the 3 directions were significantly different between barefoot and DT $(p>0.05)$. No significant differences in normalized reach distances and CoP parameters between KT and DT were observed $(p>0.05)$.

\section{DISCUSSION}

This study investigated the effects of KT and DT on static and dynamic postural control in subjects with CAI. First, OLST results demonstrated that all CoP parameters (LENGTH, AREA, and VEL) were significantly lower in the KT and DT conditions than in the barefoot condition. However, no differences between KT and DT were observed. Second, the results revealed that KT and DT have a more positive effect on balance performance in the mSEBT than the barefoot condition. Likewise, no differences between KT and DT were noted. Notably, KT and DT application potentially improves both static and dynamic postural control compared with the control group.

The CoP trajectory is the general biomechanical parameter that has been used to determine static postural control performance [35]. The shorter the LENGTH, AREA, and VEL, the better the postural control performance [11]. Generally, a key characteristic of CAI is decreased balance control. Ankle taping has proven to improve static balance performance in patients with CAI $[11,16,36]$. We found significant improvement in static postural control with KT and DT application but not in the control condition. These results were consistent with those of previous studies $[11,16,36]$. Balance improvement was represented by a decrease in the speed of postural control movements and an increase in the level of tissue stiffness around the ankle [37]. In this study, we hypothesized that ankle taping may have a positive effect on the subjects' balance regulation. Lee and Lee [36] found a positive effect of KT on providing more ankle stability to subjects while testing different static postural tasks on different surfaces and measuring the ankle' $s$ range of motion before and after tape application. They asserted that KT's elastic property might have assisted balance regulation by facilitating proprioception around the ankle joint [36]. Likewise, lateral support was provided by ankle tapes with elasticity, supporting the lateral ankle structures. Resultantly, the application of ankle tapes culminated in significantly lower postural sway compared with the control [11].

The three-directional reach distance scores during the mSEBT using DT were significantly higher than those in the control group, and those using KT significantly increased compared with those in the control group, except AM-direction scores. A systematic review by Wang et al. [18] suggested that DT and KT had advantages over other taping methods (sham taping or tension-free taping) with respect to the maximum reach distance. The foregoing outcomes may be explained by the possibility of tension application enhancing the joint position sense at the ankle joint by improving proprioception with approximately $35 \%$ elasticity. These mechanisms potentially result in increased stimulation of the cutaneous mechanoreceptors, thereby increasing the reach distance [36]. However, regarding AM-direction outcomes, only DT but not KT scores were significantly different from those in the barefoot condition. First, the KT and DT ankle-taping techniques differed. Although both tapes are elastic, we concluded that each tape should be uniquely applied to suit each property due to its distinct nature. Second, the application position differed between KT and DT. To maximize KT's tissue lifting, KT was 
predominantly applied with the tape stretched and the body part lengthened to utilize the proposed tape convolution effect [14]. Therefore, the probable reason underlying the different outcomes between the two tapes is that DT is applied with the muscles shortened, such as the plantar flexor and evertor muscles, thus further increasing ankle joint stiffness and providing more ankle joint stability.

This study used two different types of tapes, KT and DT. Although all means and standard deviations of DT CoP parameters were lower than those of KT, no significant differences during the OLST were observed. Likewise, all DT reach distance scores were higher than those of KT, and no significant differences were observed during the SEBT. The KT's primary mode of action is a neurophysiological effect that stimulates mechanoreceptors, resulting in increased sensory input and improvement of proprioception. Nonetheless, DT's primary action is a recoil effect that allows for mechanical, deceleration and load absorption $[13,14]$. Further studies are required to identify those differences, either by evaluating the proprioceptive changes at the ankle joint or the kinematic and kinetic changes during more dynamic tasks, such as running, jumping, and side-cutting tasks.

This study has certain limitations. Due to the relatively young age of the participants, the applicability of the results to older people is unknown. Moreover, it is necessary to identify the long-term impact of these tapes in future studies because, herein, we investigated the immediate effect of the elastic tapes on patients with CAI. To the best of our knowledge, there is a wide variety of taping techniques. Because the effect on the ankle joint varies depending on the taping technique, future studies should perform comparative investigation of different tape applications.

\section{CONCLUSIONS}

This study demonstrated that KT and DT had the positive effect of enhancing static and dynamic postural control in individuals with CAI during the OLST and SEBT. No significant differences were observed in OLST and SEBT parameters between KT and DT. The elastic tapes are potentially useful during the rehabilitation of people with CAI. Furthermore, our findings may guide people with CAI in the use of elastic tapes to prevent secondary ankle sprains.

\section{ACKNOWLEDGEMENTS}

This study was supported by the "Brain Korea 21 FOUR Project", the Korean Research Foundation for the Department of Physical Therapy in the Graduate School of Yonsei University.

\section{CONFLICTS OF INTEREST}

No potential conflict of interest relevant to this article was reported.

\section{AUTHOR CONTRIBUTIONS}

Conceptualization: JL, SK, IM, CY. Data curation: JL, SK. Formal analysis: JL, SK, IM, CY. Investigation: JL, SK. Methodology: JL, CY. Project administration: JL, SK, IM, CY. Resources: JL, SK, IM, CY. Supervision: CY. Visualization: JL, IM. Writing - original draft: JL, CY. Writing - review \& editing: JL, SK, IM, CY.

\section{ORCID}

Jin-seok Lim, https://orcid.org/0000-0002-9965-9619

Seo-hyun Kim, https://orcid.org/0000-0002-6978-200X

Il-young Moon, https://orcid.org/0000-0001-6457-8125

\section{REFERENCES}

1. Fong DT, Hong Y, Chan LK, Yung PS, Chan KM. A systematic review on ankle injury and ankle sprain in sports. Sports Med 2007;37(1):73-94.

2. Beynnon BD, Murphy DF, Alosa DM. Predictive factors for lateral ankle sprains: a literature review. J Athl Train 2002; 37(4):376-80.

3. Yeung MS, Chan KM, So CH, Yuan WY. An epidemiological survey on ankle sprain. Br J Sports Med 1994;28(2):112-6.

4. Freeman MA, Dean MR, Hanham IW. The etiology and prevention of functional instability of the foot. J Bone Joint Surg $\mathrm{Br}$ 1965;47(4):678-85.

5. Arnold BL, De La Motte S, Linens S, Ross SE. Ankle instability is associated with balance impairments: a meta-analysis. Med Sci Sports Exerc 2009;41(5):1048-62.

6. Bressel E, Yonker JC, Kras J, Heath EM. Comparison of static and dynamic balance in female collegiate soccer, basketball, 
and gymnastics athletes. J Athl Train 2007;42(1):42-6.

7. Hadadi M, Mousavi ME, Fardipour S, Vameghi R, Mazaheri M. Effect of soft and semirigid ankle orthoses on Star Excursion Balance Test performance in patients with functional ankle instability. J Sci Med Sport 2014;17(4):430-3.

8. Hamlyn C, Docherty CL, Klossner J. Orthotic intervention and postural stability in participants with functional ankle instability after an accommodation period. J Athl Train 2012;47(2): 130-5.

9. McKeon PO, Ingersoll CD, Kerrigan DC, Saliba E, Bennett BC, Hertel J. Balance training improves function and postural control in those with chronic ankle instability. Med Sci Sports Exerc 2008;40(10):1810-9.

10. Jackson K, Simon JE, Docherty CL. Extended use of kinesiology tape and balance in participants with chronic ankle instability. J Athl Train 2016;51(1):16-21.

11. Sarvestan J, Ataabadi PA, Svoboda Z, Kovačikova Z, Needle AR. The effect of ankle Kinesio ${ }^{\mathrm{TM}}$ taping on ankle joint biomechanics during unilateral balance status among collegiate athletes with chronic ankle sprain. Phys Ther Sport 2020;45:161-7.

12. Fayson SD, Needle AR, Kaminski TW. The effect of ankle kinesio tape on ankle muscle activity during a drop landing. J Sport Rehabil 2015;24(4):391-7.

13. Sarvestan J, Svoboda Z. Acute effect of ankle kinesio and athletic taping on ankle range of motion during various agility tests in athletes with chronic ankle sprain. J Sport Rehabil 2019;29(5):527-32.

14. McNeill W, Pedersen C. Dynamic tape. Is it all about controlling load? J Bodyw Mov Ther 2016;20(1):179-88.

15. Fazeli SH, Amiri A, Jamshidi AA, Sanjari MA, Bagheri R, Rahimi F, et al. Effect of ankle taping on postural control measures during grasp and release task in patients with chronic ankle instability. J Back Musculoskelet Rehabil 2018;31(5):881-7.

16. Kim MK, Shin YJ. Immediate effects of ankle balance taping with kinesiology tape for amateur soccer players with lateral ankle sprain: a randomized cross-over design. Med Sci Monit 2017;23:5534-41.

17. Leanderson J, Ekstam S, Salomonsson C. Taping of the ankle-the effect on postural sway during perturbation, before and after a training session. Knee Surg Sports Traumatol Arthrosc 1996;4(1):53-6.

18. Wang Y, Gu Y, Chen J, Luo W, He W, Han Z, et al. Kinesio taping is superior to other taping methods in ankle functional performance improvement: a systematic review and meta- analysis. Clin Rehabil 2018;32(11):1472-81.

19. Jelinek HF, Khalaf K, Poilvet J, Khandoker AH, Heale L, Donnan $\mathrm{L}$. The effect of ankle support on lower limb kinematics during the Y-balance test using non-linear dynamic measures. Front Physiol 2019;10:935.

20. Andreo P, Khalaf K, Heale L, Jelinek HF, Donnan L. Effects of kinesiology tape on non-linear center of mass dispersion during the Y balance test. Front Physiol 2018;9:1527.

21. Shields CA, Needle AR, Rose WC, Swanik CB, Kaminski TW. Effect of elastic taping on postural control deficits in subjects with healthy ankles, copers, and individuals with functional ankle instability. Foot Ankle Int 2013;34(10):1427-35.

22. Cortesi M, Cattaneo D, Jonsdottir J. Effect of kinesio taping on standing balance in subjects with multiple sclerosis: a pilot study. NeuroRehabilitation 2011;28(4):365-72.

23. Semple S, Esterhuysen C, Grace J. The effects of kinesio ankle taping on postural stability in semiprofessional rugby union players. J Phys Ther Sci 2012;24(12):1239-42.

24. Nakajima MA, Baldridge C. The effect of kinesio ${ }^{\circledR}$ tape on vertical jump and dynamic postural control. Int J Sports Phys Ther 2013;8(4):393-406.

25. Hettle D, Linton L, Baker JS, Donoghue 0. The effect of kinesiotaping on functional performance in chronic ankle instability-preliminary study. Clin Res Foot Ankle 2013;1(1):105.

26. Briem K, Eythörsdöttir H, Magnúsdóttir RG, Pálmarsson R, Rúnarsdöttir T, Sveinsson T. Effects of kinesio tape compared with nonelastic sports tape and the untaped ankle during a sudden inversion perturbation in male athletes. J Orthop Sports Phys Ther 2011;41(5):328-35.

27. Bicici S, Karatas N, Baltaci G. Effect of athletic taping and kinesiotaping $^{\circledR}$ on measurements of functional performance in basketball players with chronic inversion ankle sprains. Int J Sports Phys Ther 2012;7(2):154-66.

28. Gribble PA, Delahunt E, Bleakley C, Caulfield B, Docherty CL, Fourchet F, et al. Selection criteria for patients with chronic ankle instability in controlled research: a position statement of the International Ankle Consortium. J Orthop Sports Phys Ther 2013;43(8):585-91.

29. Maktouf W, Durand S, Boyas S, Pouliquen C, Beaune B. Combined effects of aging and obesity on postural control, muscle activity and maximal voluntary force of muscles mobilizing ankle joint. J Biomech 2018;79:198-206.

30. Olmsted LC, Carcia CR, Hertel J, Shultz SJ. Efficacy of the star excursion balance tests in detecting reach deficits in subjects 
with chronic ankle instability. J Athl Train 2002;37(4):501-6.

31. Hertel J, Braham RA, Hale SA, Olmsted-Kramer LC. Simplifying the star excursion balance test: analyses of subjects with and without chronic ankle instability. J Orthop Sports Phys Ther 2006;36(3):131-7.

32. Hertel J, Miller SJ, Denegar CR. Intratester and intertester reliability during the star excursion balance tests. J Sport Rehabil 2000;9(2):104-16.

33. Hadadi M, Abbasi F. Comparison of the effect of the combined mechanism ankle support on static and dynamic postural control of chronic ankle instability patients. Foot Ankle Int 2019;40(6):702-9.

34. Gribble PA, Hertel J. Considerations for normalizing measures of the star excursion balance test. Measurement Phys Educ Exerc Sci 2003;7(2):89-100.

35. Abián-Vicén J, Alegre LM, Fernández-Rodríguez JM, Lara AJ, Meana M, Aguado X. Ankle taping does not impair performance in jump or balance tests. J Sports Sci Med 2008;7(3): 350-6.

36. Lee BG, Lee JH. Immediate effects of ankle balance taping with kinesiology tape on the dynamic balance of young players with functional ankle instability. Technol Health Care 2015;23(3):333-41.

37. Paillard T, Noé F. Techniques and methods for testing the postural function in healthy and pathological subjects. Biomed Res Int 2015;2015:891390. 